\title{
Composition and Emission Rhythm of Floral Scent Volatiles from Eight Lily Cut Flowers
}

\author{
Ying Kong \\ Department of Ornamental Horticulture, College of Landscape Architecture, Beijing Forestry \\ University, Beijing 100083, China; and Beijing Radiation Center, Beijing 100875, China \\ Ming Sun \\ Department of Ornamental Horticulture, College of Landscape Architecture, Beijing Forestry \\ University, Beijing 100083, China; and Chinese National Engineering Research Center for \\ Floriculture, Beijing 100083, China \\ Hui-tang Pan \\ Department of Ornamental Horticulture, College of Landscape Architecture, Beijing Forestry \\ University, Beijing 100083, China; and Chinese National Engineering Research Center for \\ Floriculture, Beijing 100083, China \\ Qi-xiang Zhang ${ }^{1}$ \\ Department of Ornamental Horticulture, College of Landscape Architecture, Beijing Forestry \\ University, Beijing 100083, China; and Chinese National Engineering Research Center for \\ Floriculture, Beijing 100083, China
}

\begin{abstract}
AdDitional Index words. Lilium, volatile compounds, GC/MS
Abstract. Floral scents emitted from eight cultivars of cut lily flowers (Lilium) were analyzed. Floral volatiles were collected by headspace adsorption on sorbent tubes and analyzed by gas chromatography-mass spectrometry (GC/ MS) using a direct thermal desorption. Fifty volatile compounds were identified. Nine compounds were detected in all lilies, whereas 20 compounds were detected in all scented lilies. The results revealed that non-scented lilies emitted trace amounts of volatile compounds, whereas scented lilies emitted high levels of volatile compounds. Monoterpenoids and benzenoids were the dominant compound classes of volatiles emitted from scented lilies. Myrcene, $(E)$ $\beta$-ocimene, linalool, methyl benzoate, and ethyl benzoate were the major compounds of the aroma of scented lilies; 1,8-cineole was also a major compound in the two scented oriental $\times$ trumpet hybrid lilies. Scent emissions occurred in a circadian rhythm with higher levels of volatiles emitted during the night. Lilium 'Siberia' was selected as a model to investigate the source of the emissions. GC/MS analysis of four flower parts and neutral red staining revealed that tepals were the source of floral scent.
\end{abstract}

Floral fragrance is a desirable character for ornamental plants and cut flowers (Chandler and Brugliera, 2011). For instance, consumers of sweet pea (Lathyrus odoratus) consider flower aroma to be more attractive than flower shape or color (Sexton et al., 2005). However, many modern cut flowers such as rose (Rosa sp.) and carnation (Dianthus sp.) lack distinct fragrance as a result of a lack of selection for the scent trait (Clery et al., 1999; Dudareva and Pichersky, 2000). It was speculated that the vase life of cut rose was negatively correlated with fragrance, but recently it has been found that neither exogenous nor endogenous ethylene had any effect on volatile emission in roses (Borda et al., 2011).

Floral scent emission patterns often match the activity pattern of pollinators, so they are often investigated from both ecological and systematic perspectives (Meekijjaroenroj et al., 2007). Aroma emission generally starts when the flower is fully opened and peaks during periods when pollinators are most

Received for publication 21 Feb. 2012. Accepted for publication 5 Sept. 2012. This work was supported by the National Key Technology R\&D Program for the 12th Five-Year Plan (No. 2012BAD01B07) and the Fundamental Research Funds for the Central Universities (BLJD200910).

${ }^{1}$ Corresponding author. E-mail: zqx@bjfu.edu.cn. active (Dudareva and Pichersky, 2000; Jiang et al., 2011; Pichersky et al., 2006). Changes in scent emission rates between day and night have been reported in many species, which are controlled by irradiation (Helsper et al., 1998; Jakobsen and Olsen, 1994) or by an endogenous/circadian clock (Dudareva et al., 2003; Kolosova et al., 2001a; Roeder et al., 2007).

Lily is one of the most popular cut flowers in the world. Lily is also a good material to study floral scent because numerous cultivars vary in morphology and fragrance. Previous studies on the floral scent compositions of Lilium auratum (belonging to the oriental section) and Lilium 'Casa Blanca' (an oriental hybrid lily) reported that monoterpenoids and benzenoids were predominant in their aroma (Morinaga et al., 2009; OyamaOkubo et al., 2011). In L. auratum, (E)- $\beta$-ocimene, methyl benzoate, isoeugenol, and linalool were the major compounds, whereas in Lilium 'Casa Blanca', linalool, cis-ocimene, benzyl alcohol, and isoeugenol predominated. Furthermore, volatile emissions reached their maximum at night. It has been speculated that the nocturnal bias for the emission pattern of L. auratum is an adaptation toward achieving male reproductive success by attracting nocturnal pollinators. Therefore, the first objective of this study is to identify the volatile compounds 
emitted from scented lily cut flowers and to determine whether non-scented lily cut flowers emit any volatiles. The second objective is to determine whether cut lily flowers also show nocturnal emission patterns.

It is known that in many flowers, most of their fragrance is produced by coralla or petals (Dobson et al., 1990). However, other flower parts can also play an important role in scent emission. For example, stamens and stigma also have scent contributions (Bergström et al., 1995). Hence, the third objective of this study is to identify the parts of the flower from where volatiles were emitted.

\section{Materials and Methods}

Plant materials. Fresh lily cut flowers of eight cultivars (buds and flowers included) were purchased from a morning flower market. Lilies belonged to the groups of oriental hybrids ('Siberia', 'Marco Polo', and 'Sorbonne'), OT (oriental $\times$ trumpet) hybrids ('Yelloween' and 'Conca d'Or'), longiflorum hybrids ('White Heaven'), asiatic hybrids ('Tresor'), and LA (longiflorum $\times$ asiatic) hybrids ('Ceb Dazzle'). They are popular flowers in China, and their characteristics are summarized in Table 1. Flowers were kept in an indoor environment $\left(26 \pm 1{ }^{\circ} \mathrm{C}\right.$, photoperiod $=0800$ to $2000 \mathrm{HR}$ light, 2000 to $0800 \mathrm{HR}$ dark) (Oyama-Okubo et al., 2011). Stems of cut flowers were placed in deionized water. Anthers were removed after anthesis. Flowers in the basal part of the inflorescences were used for sampling. Experiments were conducted between late Sept. and early Oct. 2011.

Floral scent Collection. Volatile compounds emitted from cut flowers were collected using a static headspace adsorption method. Before sampling, sorbent tubes (CAMSCO, Houston, TX) filled with $200 \mathrm{mg}$ Tenax GR (60/80 mesh; Alltech, Deerfield, IL) were pre-conditioned by flowing helium through at $100 \mathrm{~mL} \cdot \mathrm{min}^{-1}$ while heated at $270{ }^{\circ} \mathrm{C}$ for $120 \mathrm{~min}$ to ensure the removal of residual compounds. To minimize the effects of diurnal fluctuations, detached flowers were first sampled during the day (0900 to $1000 \mathrm{HR}$ ). Afterward, their pedicels were placed in tap water until a second sampling was conducted during the night (2100 to $2200 \mathrm{HR}$ ). Each lily flower (including the pedicel but without anthers) was enclosed in an oven bag (406 × 444 mm; Reynolds, Richmond, VA) 1 d after anthesis.

After complete extraction of the pre-existing air, the bag was filled with $1 \mathrm{~L}$ of air purified by activated carbons at a flow rate of $1 \mathrm{~L} \cdot \mathrm{min}^{-1}$. Then the bag was enclosed for $10 \mathrm{~min}$. Subsequently, the air containing volatile compounds was extracted using a mini-pump (GSP-300FT-2; Gastec Corp., Kanagawa,
Japan) at a flow rate of $200 \mathrm{~mL} \cdot \mathrm{min}^{-1}$ for $5 \mathrm{~min}$, and the volatiles were trapped in sorbent tubes. Oven bags without flowers were used as a control. The procedure was repeated three or four times. After sampling, the sorbent tubes were kept at $-20{ }^{\circ} \mathrm{C}$ until analysis (Jürgens et al., 2009).

IDENTIFICATION OF VOLATILE-EMITTING FLOWER PARTS. Lilium 'Siberia' was used to analyze volatile compounds emissions from different flower parts. First, volatiles emitted from the entire flower were sampled at 2100 to $2200 \mathrm{HR}$. Then, the entire flower was immediately divided into four parts. Volatile compounds emitted from tepals, stamens, anthers, and others (ovary and pistils coupled with pedicel) were collected using the same sampling method described previously.

Histochemical staining. Neutral red stain was used to identify the location of osmophores responsible for volatile emission (Effmert et al., 2005). To localize the areas containing osmophores, Lilium 'Siberia' flowers (anthers removed) were submerged in a solution of 1:10,000 neutral red:tap water $(\mathrm{w} / \mathrm{m})$. Staining was performed in the dark for $24 \mathrm{~h}$ (Rohrbeck et al., 2006; Wright and Schiestl, 2009). Peduncles were sealed with wax to ensure that the stain was taken through the osmophores instead of the cut tissue. Excess stain was removed by rinsing the flowers with tap water. Photographs of pre- and post-stained flowers were taken using a digital camera (FinePix F50; Fujifilm, Tokyo, Japan).

GAS CHROMATOGRAPHY-MASS SPECTROSCOPY ANALYSIS. The tubes containing the volatiles were desorbed in an automatic thermal desorber (Turbo Matrix 650; PerkinElmer, Waltham, $\mathrm{MA})$ at $260^{\circ} \mathrm{C}$ for $10 \mathrm{~min}$. Helium flow rate was $1.5 \mathrm{~mL} \cdot \mathrm{min}^{-1}$. The desorbed volatile compounds were first trapped in the cold $\operatorname{trap}\left(-25^{\circ} \mathrm{C}\right)$, which was then quickly heated to $300^{\circ} \mathrm{C}$ at a rate of $40{ }^{\circ} \mathrm{C} \cdot \mathrm{s}^{-1}$ and then injected into the GC through a capillary transfer line at $250{ }^{\circ} \mathrm{C}$. Analysis was performed using a GC (Clarus 600; PerkinElmer) coupled to a MS (Clarus 600T; PerkinElmer). The GC was fitted with a DB-5MS fused-silica capillary column $(30 \mathrm{~m} \times 0.25 \mathrm{~mm} \times 0.25 \mathrm{~mm}$; Agilent Technologies, Santa Clara, CA). The GC oven temperature was programmed at $40{ }^{\circ} \mathrm{C}$ for $2 \mathrm{~min}$, then the temperature was increased at a rate of $6{ }^{\circ} \mathrm{C} \cdot \mathrm{min}^{-1}$ to $180{ }^{\circ} \mathrm{C}$, followed by an increase at $15^{\circ} \mathrm{C} \cdot \mathrm{min}^{-1}$ to $270{ }^{\circ} \mathrm{C}$, and finally held at $270{ }^{\circ} \mathrm{C}$ for $3 \mathrm{~min}$. The mass spectra were taken in electron ionization at $70 \mathrm{eV}$, and the mass scan range was 30 to $500 \mathrm{~m} / \mathrm{z}$.

TurboMass 5.4.2 GC/MS software (PerkinElmer) was used for the compound analysis. Compounds were identified by matching the mass spectra with NIST 08 library (National Institute of Standards and Technology, Gaithersburg, MD) and retention index, and their identities were confirmed by comparing retention index and mass spectra with those in the

Table 1. Floral characteristics of eight lily cut flowers used for floral scent analysis.

\begin{tabular}{lllll}
\hline Cultivar & Flower color & Aroma level $^{z}$ & Description of floral scent & Dsiatic hybrids \\
\hline Tresor & Orange & Non-scented & - & LA (longiflorum $\times$ asiatic) hybrids \\
Ceb Dazzle & Yellow & Non-scented & & Longiflorum hybrids \\
White Heaven & White & Light-scented & Fresh, herbaceous & Oriental hybrids \\
Marco Polo & Pink & Strong-scented & Fragrant, floral, perfume & Oriental hybrids \\
Siberia & White & Strong-scented & Fragrant, sweet, flowery & Oriental hybrids \\
Sorbonne & Pink & Strong-scented & Fragrant, floral, perfume & OT (oriental $\times$ trumpet) hybrids \\
Conca d'Or & Yellow & Strong-scented & Fragrant, sweet, musky & OT (oriental $\times$ trumpet) hybrids \\
Yelloween & Yellow & Strong-scented & Fragrant, floral, musky &
\end{tabular}

${ }^{\mathrm{z} S c e n t e d}$ by day. 


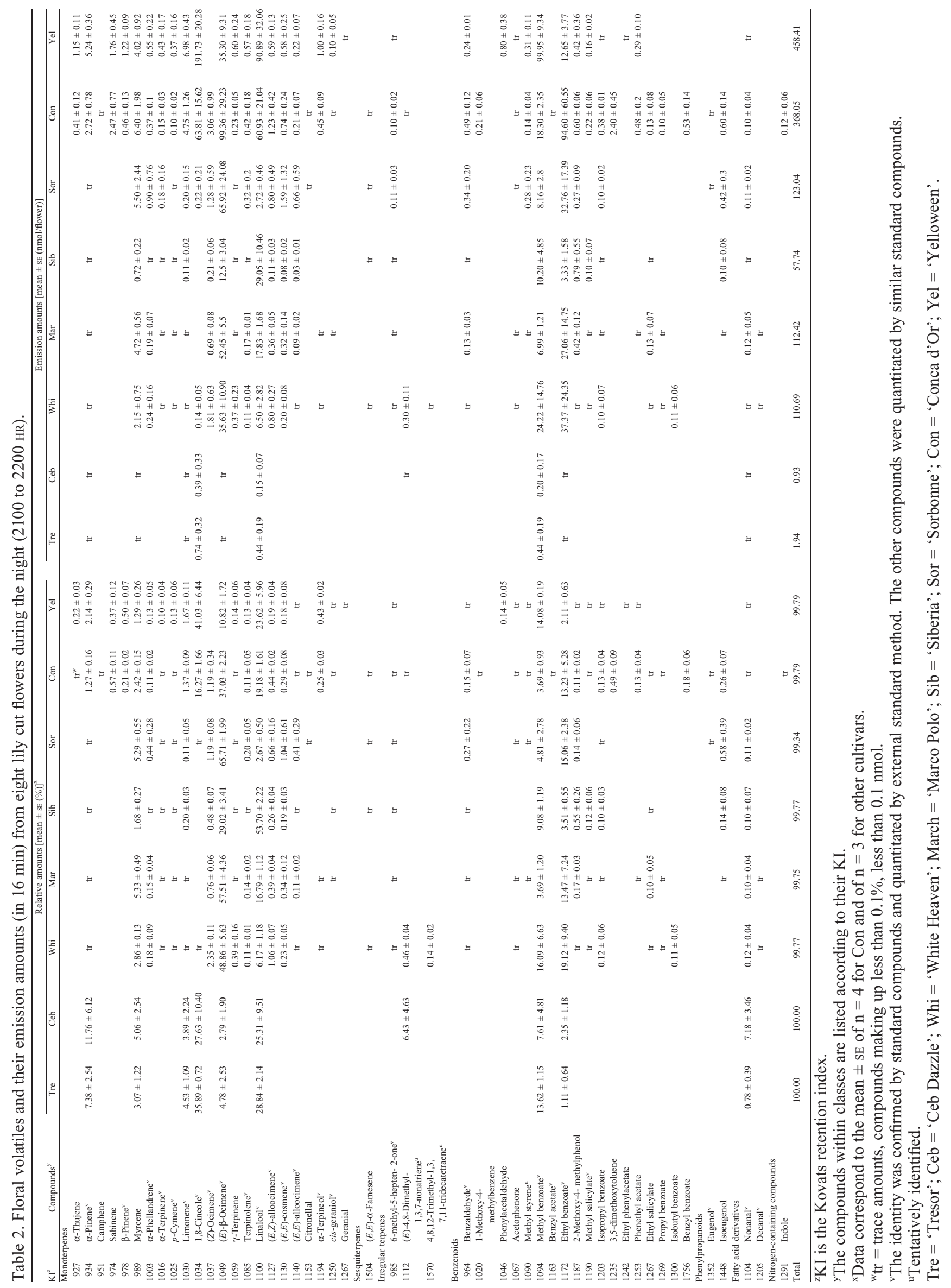


Pherobase database (El-Sayed, 2012) or authentic standards when available (Proffit and Johnson, 2009). Known amounts of monoterpenoids, benzenoids, and fatty acid derivatives were used for quantification by an external standard method (Balao et al., 2011). Peak areas were normalized as percentage and used to determine the relative amounts of the volatiles.

Data ANalysis. The emission abundances of major compounds (greater than $1 \%$ in any cultivar) were analyzed by principal component analysis [PCA (on a covariance matrix)] using SPSS (Version 17.0; IBM Corp., Armonk, NY) to identify differences in scent composition among the eight lily cultivars analyzed.

\section{Results}

COMPOSITION OF FLORAL SCENT EMITTED FROM EIGHT CUT LILY FLOWERS DURING THE NIGHT. We identified 50 volatile compounds, representing more than $99 \%$ of the total nocturnal emission of the flowers. These volatiles grouped by their biochemical synthesis pathways are described in Table 2 (Knudsen et al., 1993). Most of these compounds are commonly found in floral scents. The number of volatile compounds detected in the flowers varied from nine ('Tresor') to 44 compounds ('Conca d'Or'). There were nine compounds emitted from all lily cut flowers and 20 compounds emitted from all scented lily cut flowers.

A wide variety of volatile compounds were identified in scented lily cut flowers, including monoterpenoids, benzenoids, sesquiterpenoids, phenylpropanoids, fatty acid derivatives, and $\mathrm{N}$-containing compounds. In scented lilies, monoterpenoids accounted for $62.59 \%$ to $85.95 \%$, and benzenoids represented $13.47 \%$ to $35.59 \%$ of total volatile emission amounts. Most of these compounds were found at low emission amounts. Only a few compounds represented more than $1 \%$ of total emissions. Myrcene, $(E)$ - $\beta$-ocimene, linalool, methyl benzoate, and ethyl benzoate were detected at high emission amounts in all scented lily cut flowers, accounting for $51.90 \%$ to $96.99 \%$ of total emissions (Fig. 1). Interestingly, 1,8-cineole was found at relatively high levels in OT hybrid lilies ('Yelloween' and 'Conca d'Or').

On the other hand, in non-scented 'Tresor' and 'Ceb Dazzle', the number of volatile compounds was significantly lower than that in scented lilies. 1,8-Cineole, linalool, methyl benzoate, and $\alpha$-pinene were the major aroma compounds of non-scented lilies. However, these volatiles were emitted at very low levels.

Principal Component analysis of Floral SCENT EMissions. Principal component analysis was performed to categorize the cultivars based on their fragrance compositions. PC1 and PC2, the first two principal components, were obtained by the analysis of the 11 major compounds (covariance matrix) and explained $50.77 \%$ and $37.25 \%$ of the variation, respectively (Fig. 2). The loading analysis showed that the eight cultivars were separated by a few variables.

The positions of the non-scented lilies ('Tresor' and 'Ceb Dazzle') almost overlapped in the plot, which may be explained by their low scent emissions. Two of the oriental hybrid lilies, 'Marco Polo' and 'Sorbonne', had similar positions. 'Siberia' is also an oriental hybrid lily, but its lower emissions of $(E)$ $\beta$-ocimene and higher emissions of linalool separated it from 'Marco Polo' and 'Sorbonne'. 'Yelloween' and 'Conca d'Or' were separated distinctly from each other and from the other

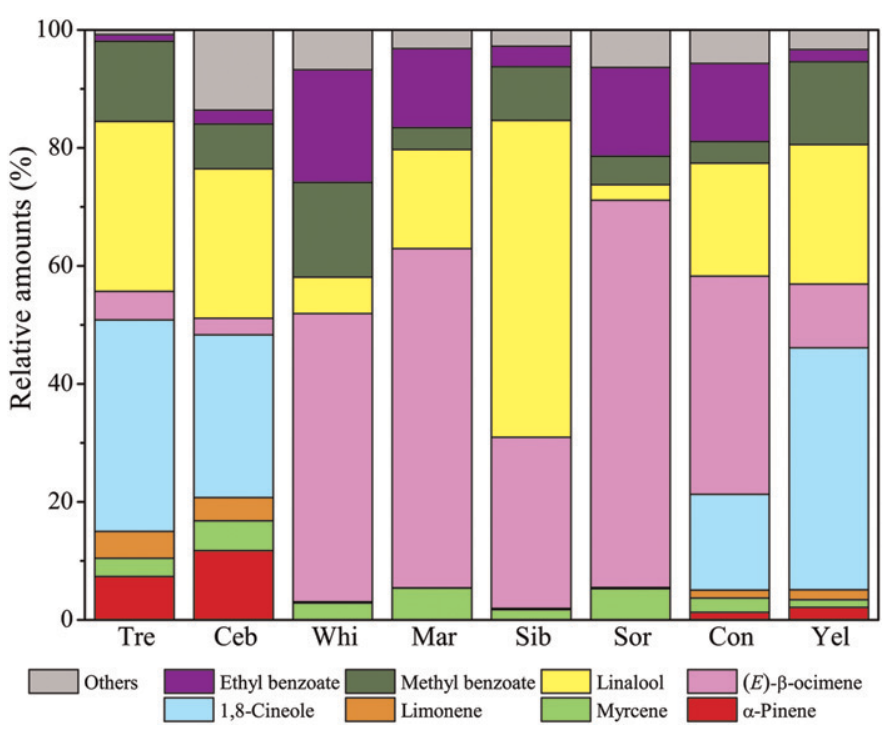

Fig. 1. Major compounds of floral scent emitted from eight lily cut flowers during the night (2100 to 2200 HR): 'Tresor' (Tre), 'Ceb Dazzle' (Ceb), 'White Heaven' (Whi), 'Marco Polo' (Mar), 'Siberia' (Sib), 'Sorbonne' (Sor), 'Conca d'Or' (Con), and 'Yelloween' (Yel).

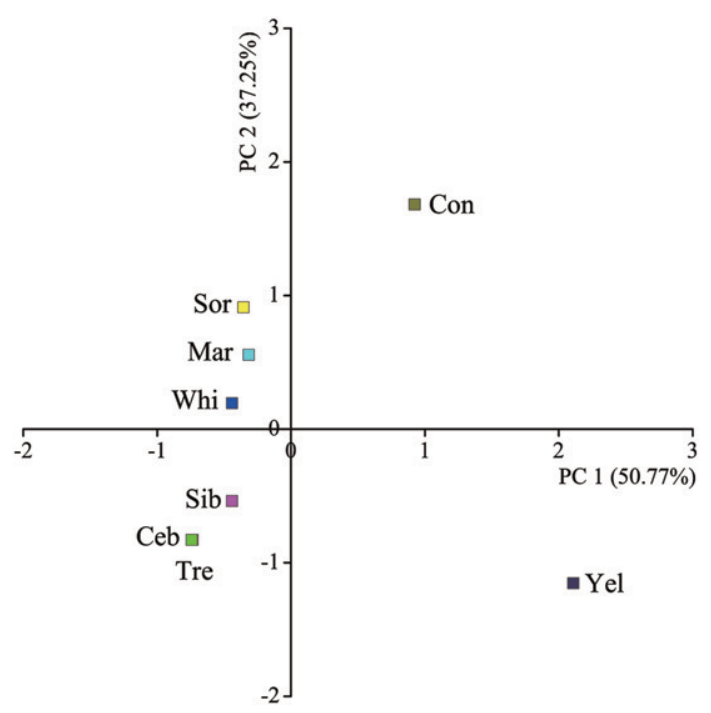

Fig. 2. Principal component analysis of the abundance of 11 major compounds (greater than $1 \%$ in any cultivar on a covariance matrix) in the floral scent separated the eight lily cut flowers: 'Tresor' (Tre), 'Ceb Dazzle' (Ceb), 'White Heaven' (Whi), 'Marco Polo' (Mar), 'Siberia' (Sib), 'Sorbonne' (Sor), 'Conca d'Or' (Con), and 'Yelloween' (Yel). The first axis was highly correlated with the amounts of $\alpha$-pinene $(0.987)$, limonene $(0.984), 1,8$-cineole $(0.967)$, linalool $(0.966)$, and methyl benzoate $(0.897)$. The second axis was correlated with the amounts of $(Z)$-ocimene $(0.896),(E)$ - $\beta$-ocimene $(0.896)$, and ethyl benzoate $(0.886)$.

lilies. Compared with 'Conca d'Or', 'Yelloween' emitted more 1,8-cineole and methyl benzoate but less $(E)$ - $\beta$-ocimene. The position of 'White Heaven' was close to 'Marco Polo' and 'Sorbonne', which may be caused by their similar scent compositions.

DAY/NIGHT SCENT EMISSION RHYTHMiCITY. The emissions of scent volatiles from all eight cultivars exhibited day/night rhythmic patterns. The amounts of volatile compounds emitted during the night were significantly higher than those emitted 
during the day (Fig. 3). Even the non-scented cultivars Tresor and Ceb Dazzle, which emitted almost none during the day, emitted small amounts of volatile compounds during the night. In scented lilies, both total emissions and major component emissions increased significantly at night.

Volatile-emitting flower Parts. Composition and emission amounts of volatiles emitted from tepals were similarly with that emitted from the whole flower (anther removed). Other floral parts (stamens, anthers, ovary and pistils coupled with pedicel) emitted little or no volatiles or not detected resulting from the short sampling time or low weights. This result agrees with human sensory impressions. Tepals smelled sweet and fragrant, similar to the whole flower, but other parts had no scent.

Neutral red staining confirmed these observations (Fig. 4). Treated flowers showed staining in tepals, stigma, and the apical margin of the filament. Staining was most intense in tepals (both adaxial and abaxial) and nectar glands, whereas vascular tissues were only slightly colored. Interestingly, the

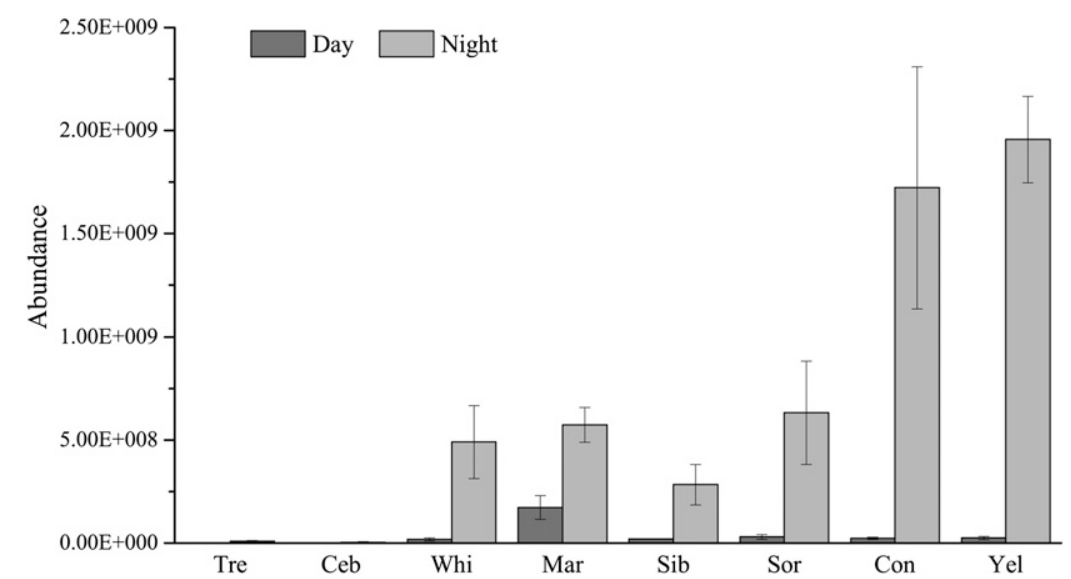

Fig. 3. Comparison of emission amounts of eight lily cut flowers during the day (0900 to $1000 \mathrm{HR}$ ) and night (2100 to 2200 HR): 'Tresor' (Tre), 'Ceb Dazzle' (Ceb), 'White Heaven' (Whi), 'Marco Polo' (Mar), 'Siberia' (Sib), 'Sorbonne' (Sor), 'Conca d'Or' (Con), and 'Yelloween' (Yel). Data correspond to the mean \pm SE of $n=4$ for Con and of $n=3$ for other cultivars.

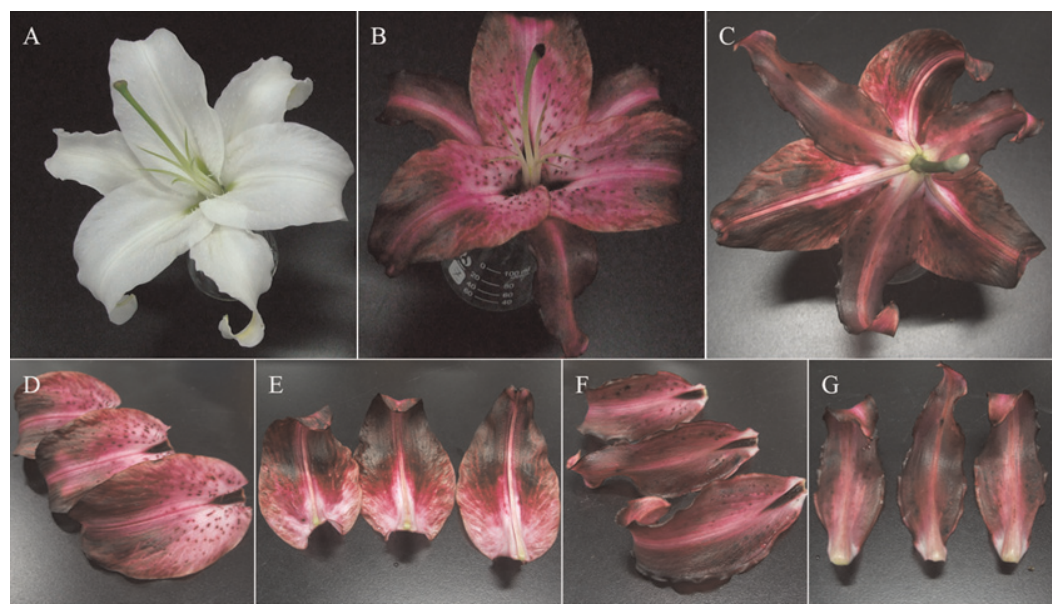

Fig. 4. Neutral red staining of detached Lilium 'Siberia' flowers (anthers removed). Flowers were immersed in an aqueous neutral red solution $(0.01 \%$ in dark, $24 \mathrm{~h})$ followed by rinsing with tap water. (A) Control. B-G are from stained flowers; (B) frontal view of the whole flower, (C) dorsal view of the whole flower, (D) frontal view of inner tepals, (E) dorsal view of inner tepals, (F) frontal view of outer tepals, $(\mathbf{G})$ dorsal view of outer tepals. curved parts of inner tepals showed a deeper color. However, in outer tepals, no obvious differences in staining were observed.

\section{Discussion}

In this article, we reported the compositions of floral scent emitted from eight cultivars of lily cut flowers. Volatile compounds emitted from scented and non-scented flowers were qualitatively and quantitatively distinct. Non-scented lilies (asiatic and LA hybrid) emitted only trace amounts and are unable to be detected by the human nose. Scented lilies emitted high amounts of volatiles. In previous and our studies, we found tinalool, $(E)$ - $\beta$-ocimene, and other monoterpenes. They smell fragrant and sweet, which may be the reason for the odor characteristic of scented oriental hybrid lilies. The two scented OT 作 cineole, etc. The first two components explain their fragrant smell and the last may explain their musky characteristic. Contrary to our expectations, 'White Heaven', a longiflorum hybrid lily, had a scent composition similar to those of some oriental hybrid lilies.

Like other hereditary traits, volatile scent composition also reflects taxonomic affinities (Nogueira et al., 2001). Many non-scented or little-scented Lilium species have been used to breed asiatic hybrid lilies, so these progenitors may have passed this trait down to hybrid progenies such as 'Tresor' and 'Ceb Dazzle'. Many oriental hybrid lilies have powerful, sweet fragrances, which originated from their fragrant lily ancestors such as L. auratum, L. speciosum, L. japonicum, etc. In our study, the two OT hybrid lilies not only emitted high levels of $(E)$ - $\beta$-ocimene and linalool, but also high levels of 1,8-cineole and benzenoid esters. Interestingly, in some trumpet lilies such as L. sulphureum, 1,8cineole and methyl benzoate are the major components (Kong et al., in press). Thus, scented OT hybrid lilies may have inherited their scent traits from both progenitors. Nevertheless, because the family tree of 'White Heaven' is not clear, further research is needed to explain the composition of its scent.

Generally speaking, biosynthesis of volatile compounds result from a combination of related enzymes and substrate availability. We have found monoterpenoids and benzenoids were the predominant compound classes in the aromas of scented lilies. Geranyl pyrophosphate (or neryl diphosphate) is the precursor of monoterpenes (Degenhardt et al., 2009), and the monoterpene synthase involved in the monoterpenoid biosynthesis determines the products. Because all scented lilies emitted high levels of monoterpenoids, the divergence in monoterpene products mainly depends on the responsible monoterpene synthase. For example, if the enzyme is $(E)$ - $\beta$-ocimene synthase, a high proportion 
of $(E)$ - $\beta$-ocimene will be generated, and if the enzyme is 1,8 cineole synthase, the main product will be 1,8-cineole. Other minor monterpenoids are catalyzed as minor products, because many monoterpene synthases can catalyze multiproducts from a single substrate (Degenhardt et al., 2009). On the other hand, the biosynthesis of methyl benzoate, which is catalyzed by benzoic acid carboxyl methyltransferase or benzoic/salicylic acid carboxyl methyltransferase, may be regulated by the availability of the benzoic acid (Dudareva et al., 2000). It is found that ethyl benzoate can be generated as minor products by alcohol acyltransferases catalyzing alcohol mix (Günther et al., 2011). However, the biosynthesis of ethyl benzoate is still not clear, and scented lily with high ethyl benozate emission may be a good material to study it.

We found that all eight lily cut flower cultivars exhibited nocturnal emission patterns. Similar emission rhythmicity has also been observed in other scented Lilium species. L. auratum, L. japonicum, and Lilium 'Casa Blanca' have shown maximum emission levels at night (Morinaga et al., 2009; Oyama-Okubo et al., 2011; Yokota and Yahara, 2012). The same scent emission patterns were also observed in L. sulphureum and L. leucanthum (unpublished data). The high nocturnal emission levels of these flowers should be taken into account when looking for a space to place them, because many people suffer from intolerance to strong fragrances. In addition, some volatile compounds can have effects on the cardiovascular system. For example, a high dose of 1,8-cineole can elicit hypotension (Lahlou et al., 2002). Nevertheless, research in this area is rare.

The analysis of volatiles emitted from different parts of Lilium 'Siberia' flowers revealed that tepals are the major source of floral scent. It is the first report of scent emission from tepals only in lily. The result agrees with previous reports identifying petals as the main source of scent (Bergougnoux et al., 2007; Pichersky et al., 1994; Rohrbeck et al., 2006). Results of neutral red staining of lily flower indicated that different tepal parts may contribute differently to scent emission. Similar results have also been found in flowers of Mirabilis alba and Stephanotis floribunda (Effmert et al., 2005; Rohrbeck et al., 2006). It is considered that spatial differences on scent emission within a flower (gynoecium, androecium, perianth) are quite common, and emitting tissue sometimes comprises only certain parts of a floral organ (Effmert et al., 2006). Early researchers discovered that the epidermis cells of the flower are the site of volatile biosynthesis and diffusion (Dudareva et al., 2004; Effmert et al., 2005; Pichersky et al., 2006). Depending on the species and floral morphology, both adaxial and abaxial epidermis cells (Nicotiana suaveolens and Clarkia breweri), or only adaxial epidermis cells (Antirrhinum majus and $S$. floribunda), contain the enzymes responsible for scent production (Bergougnoux et al., 2007; Kolosova et al., 2001b; Roeder et al., 2007; Rohrbeck et al., 2006). Lilium 'Siberia' has individual flowers, and visitors can reach flowers freely, no matter whether the scent is emitted from the adaxial and/or abaxial epidermis. Because both adaxial and abaxial tepals were stained heavily, we speculated that both adaxial and abaxial tepals may be the major emission area, so that the volatile compounds can be synthesized and diffused quickly to the atmosphere in all spacial directions.

The results of this study may lead to a new way of conducting genetics-based fragrance research. This approach would involve the isolation and characterization of the enzymes and genes involved in volatile biosynthesis followed by finding out whether substrate or enzyme affects scent production.

\section{Literature Cited}

Balao, F., J. Herrera, S. Talavera, and S. Dötterl. 2011. Spatial and temporal patterns of floral scent emission in Dianthus inoxianus and electroantennographic responses of its hawkmoth pollinator. Phytochemistry 72:601-609.

Bergougnoux, V., J.-C. Caissard, F. Jullien, J.-L. Magnard, G. Scalliet, J. Cock, P. Hugueney, and S. Baudino. 2007. Both the adaxial and abaxial epidermal layers of the rose petal emit volatile scent compounds. Planta 226:853-866.

Bergström, G., H.E.M. Dobson, and I. Groth. 1995. Spatial fragrance patterns within the flowers of Ranunculus acris (Ranunculaceae). Plant Syst. Evol. 195:221-242.

Borda, A.M., D.G. Clark, D.J. Huber, B.A. Welt, and T.A. Nell. 2011. Effects of ethylene on volatile emission and fragrance in cut roses: The relationship between fragrance and vase life. Postharvest Biol. Technol. 59:245-252.

Chandler, S. and F. Brugliera. 2011. Genetic modification in floriculture. Biotechnol. Lett. 33:207-214.

Clery, R.A., N.E. Owen, and S.F. Chambers. 1999. An investigation into the scent of carnations. J. Essential Oil Res. 11:355-359.

Degenhardt, J., T.G. Köllner, and J. Gershenzon. 2009. Monoterpene and sesquiterpene synthases and the origin of terpene skeletal diversity in plants. Phytochemistry 70:1621-1637.

Dobson, H.E.M., G. Bergström, and I. Groth. 1990. Differences in fragrance chemistry between flower parts of Rosa rugosa Thunb. (Rosaceae). Isr. J. Bot. 39:143-156.

Dudareva, N., L.M. Murfitt, C.J. Mann, N. Gorenstein, N. Kolosova, C.M. Kish, C. Bonham, and K. Wood. 2000. Developmental regulation of methyl benzoate biosynthesis and emission in snapdragon flowers. Plant Cell 12:949-961.

Dudareva, N., D. Martin, C.M. Kish, N. Kolosova, N. Gorenstein, J. Faldt, B. Miller, and J. Bohlmann. 2003. (E)- $\beta$-ocimene and myrcene synthase genes of floral scent biosynthesis in snapdragon: Function and expression of three terpene synthase genes of a new terpene synthase subfamily. Plant Cell 15:1227-1241.

Dudareva, N. and E. Pichersky. 2000. Biochemical and molecular genetic aspects of floral scents. Plant Physiol. 122:627-633.

Dudareva, N., E. Pichersky, and J. Gershenzon. 2004. Biochemistry of plant volatiles. Plant Physiol. 135:1893-1902.

Effmert, U., D. Buss, D. Rohrbeck, and B. Piechulla. 2006. Localization of the synthesis and emission of scent compounds within the flower, p. 105-124. In: Dudareva, N. and E. Pichersky (eds.). Biology of floral scent. CRC Press, Boca Raton, FL.

Effmert, U., J. Große, U.S.R. Röse, F. Ehrig, R. Kägi, and B. Piechulla. 2005. Volatile composition, emission pattern, and localization of floral scent emission in Mirabilis jalapa (Nyctaginaceae). Amer. J. Bot. 92:2-12.

El-Sayed, A.M. 2012. The Pherobase: Database of pheromones and semiochemicals. 5 Sept. 2012. <http://www.pherobase.com>.

Günther, C.S., C. Chervin, K.B. Marsh, R.D. Newcomb, and E.J.F. Souleyre. 2011. Characterisation of two alcohol acyltransferases from kiwifruit (Actinidia spp.) reveals distinct substrate preferences. Phytochemistry 72:700-710.

Helsper, J.P.F.G., J.A. Davies, H.J. Bouwmeester, A.F. Krol, and M.H. van Kampen. 1998. Circadian rhythmicity in emission of volatile compounds by flowers of Rosa hybrida L. cv. Honesty. Planta 207:88-95.

Jakobsen, H.B. and C.E. Olsen. 1994. Influence of climatic factors on emission of flower volatiles in situ. Planta 192:365-371.

Jiang, Y.F., X.L. Chen, H. Lin, F. Wang, and F. Chen. 2011. Floral scent in wisteria: Chemical composition, emission pattern, and regulation. J. Amer. Soc. Hort. Sci. 136:307-314.

Jürgens, A., A.M. El-Sayed, and D.M. Suckling. 2009. Do carnivorous plants use volatiles for attracting prey insects? Funct. Ecol. 23:875-887. 
Knudsen, J.T., L. Tollsten, and L.G. Bergström. 1993. Floral scents-A checklist of volatile compounds isolated by head-space techniques. Phytochemistry 33:253-280.

Kolosova, N., N. Gorenstein, C.M. Kish, and N. Dudareva. 2001a. Regulation of circadian methyl benzoate emission in diurnally and nocturnally emitting plants. Plant Cell 13:2333-2347.

Kolosova, N., D. Sherman, D. Karlson, and N. Dudareva. $2001 \mathrm{~b}$. Cellular and subcellular localization of $S$-adenosyl-L-methionine: benzoic acid carboxyl methyltransferase, the enzyme responsible for biosynthesis of the volatile ester methylbenzoate in snapdragon flowers. Plant Physiol. 126:956-964.

Kong Y., M. Sun, H.T. Pan, Q.X. Zhang, C.X. Ai, and Y.H. Wang. Floral scent composition of Lilium sulphureum Baker apud Hook (Liliaceae). Chemistry of Natural Compounds. (In press.)

Lahlou, S., A.F. Figueiredo, P.J. Magalhaes, and J.H. Leal-Cardoso. 2002. Cardiovascular effects of 1,8-cineole, a terpenoid oxide present in many plant essential oils, in normotensive rats. Can. J. Physiol. Pharmacol. 80:1125-1131.

Meekijjaroenroj, A., J.-M. Bessière, and M.-C. Anstett. 2007. Chemistry of floral scents in four Licuala species (Arecaceae). Flavour Fragrance J. 22:300-310.

Morinaga, S.-I., Y. Kumano, A. Ota, R. Yamaoka, and S. Sakai. 2009. Day-night fluctuations in floral scent and their effects on reproductive success in Lilium auratum. Popul. Ecol. 51:187-195.

Nogueira, P.C.L., V. Bittrich, G.J. Shepherd, A.V. Lopes, and A.J. Marsaioli. 2001. The ecological and taxonomic importance of flower volatiles of Clusia species (Guttiferae). Phytochemistry $56: 443-452$.

Oyama-Okubo, N., M. Nakayama, and K. Ichimura. 2011. Control of floral scent emission by inhibitors of phenylalanine ammonia-lyase in cut flower of Lilium cv. 'Casa Blanca'. J. Jpn. Soc. Hort. Sci. 80:190-199.

Pichersky, E., J.P. Noel, and N. Dudareva. 2006. Biosynthesis of plant volatiles: Nature's diversity and ingenuity. Science 311:808-811.

Pichersky, E., R.A. Raguso, E. Lewinsohn, and R. Croteau. 1994. Floral scent production in Clarkia (Onagraceae) (I. Localization and developmental modulation of monoterpene emission and linalool synthase activity). Plant Physiol. 106:1533-1540.

Proffit, M. and S.D. Johnson. 2009. Specificity of the signal emitted by figs to attract their pollinating wasps: Comparison of volatile organic compounds emitted by receptive syconia of Ficus sur and F. sycomorus in Southern Africa. S. Afr. J. Bot. 75:771-777.

Roeder, S., A.-M. Hartmann, U. Effmert, and B. Piechulla. 2007. Regulation of simultaneous synthesis of floral scent terpenoids by the 1,8-cineole synthase of Nicotiana suaveolens. Plant Mol. Biol. 65:107-124.

Rohrbeck, D., D. Buss, U. Effmert, and B. Piechulla. 2006. Localization of methyl benzoate synthesis and emission in Stephanotis floribunda and Nicotiana suaveolens flowers. Plant Biol. 8:615-626.

Sexton, R., A.P. Stopford, W.T. Moodie, and A.E.A. Porter. 2005. Aroma production from cut sweet pea flowers (Lathyrus odoratus): The role of ethylene. Physiol. Plant. 124:381-389.

Wright, G.A. and F.P. Schiestl. 2009. The evolution of floral scent: The influence of olfactory learning by insect pollinators on the honest signalling of floral rewards. Funct. Ecol. 23:841-851.

Yokota, S. and T. Yahara. 2012. Pollination biology of Lilium japonicum var. abeanum and var. japonicum: Evidence of adaptation to the different availability of diurnal and nocturnal pollinators. Plant Species Biol. 27:96-105. 